\title{
Estimating the Spins of Stellar-Mass Black Holes by Fitting Their Continuum Spectra
}

\section{Citation}

Narayan, Ramesh, Jeffrey E. McClintock, Rebecca Shafee, Ye-Fei Yuan, Xiang-Dong Li, and Dong Lai. 2008. "Estimating the Spins of Stellar-Mass Black Holes by Fitting Their Continuum Spectra." In the Proceedings of the International Conference on Astrophysics of Compact Objects, Huangshan City, China, July 1-7, 2007. doi:10.1063/1.2840411.

\section{Published Version}

doi:10.1063/1.2840411

\section{Permanent link}

http://nrs.harvard.edu/urn-3:HUL.InstRepos:28485583

\section{Terms of Use}

This article was downloaded from Harvard University's DASH repository, and is made available under the terms and conditions applicable to Other Posted Material, as set forth at http:// nrs.harvard.edu/urn-3:HUL.InstRepos:dash.current.terms-of-use\#LAA

\section{Share Your Story}

The Harvard community has made this article openly available.

Please share how this access benefits you. Submit a story.

\section{Accessibility}




\title{
Estimating the Spins of Stellar-Mass Black Holes by Fitting Their Continuum Spectra
}

\author{
Ramesh Narayan, Jeffrey E. McClintock and Rebecca Shafee \\ Harvard-Smithsonian Center for Astrophysics, 60 Garden St., Cambridge, MA 02138, USA
}

\begin{abstract}
We have used the Novikov-Thorne thin disk model to fit the continuum X-ray spectra of three transient black hole X-ray binaries in the thermal state. From the fits we estimate the dimensionless spin parameters of the black holes to be: $4 \mathrm{U} 1543-47, a_{*} \equiv a / M=0.7-0.85$; GRO J1655-40, $a_{*}=0.65-0.8$; GRS $1915+105, a_{*}=0.98-1$. We plan to expand the sample of spin estimates to about a dozen over the next several years. Some unresolved theoretical issues are briefly discussed.
\end{abstract}

Keywords: accretion disk; black hole; spin; X-ray binary

PACS: 04.; 97.60.Lf; 97.80.Jp

\section{INTRODUCTION}

Currently, about 40 black hole (BH) X-ray binaries are known in the Milky Way and Local Group galaxies. The masses of 21 of these BHs have been measured by observing the dynamics of their binary companion stars (McClintock \& Remillard 2006; Orosz et al. 2007). Having measured the mass $M$ of a BH, the logical next step is obviously to measure the dimensionless spin parameter, $a_{*} \equiv a / M=c J / G M^{2}$, where $J$ is the angular momentum of the $\mathrm{BH}$. Indeed, $a_{*}$ is arguably more interesting and important than $M$. Mass merely sets the scale of a $\mathrm{BH}$, whereas spin is an intrinsic parameter that determines the geometry of space-time in the vicinity of the hole.

Unfortunately, spin is much harder to measure than mass. The effects of spin are revealed only in the regime of strong gravity close to the hole, where the sole probe available to us is the accreting gas. Thus, in order to measure spin, we must make accurate observations of the radiation emitted by the inner regions of the accretion disk, and we must have a reliable model of the emission. Until recently, there was no credible measurement of $\mathrm{BH}$ spin.

The situation has now changed. Following up on the pioneering work of Zhang, Cui \& Chen (1997), our group recently estimated the spin parameters of three stellarmass BHs (Shafee et al. 2006; McClintock et al. 2006): GRO J1655-40, 4U 1543-47, and GRS 1915+105. These spin estimates (Table 1) were obtained by modeling the continuum X-ray spectra of the accretion disks surrounding the BHs. Independently, Davis, Done \& Blaes (2006) estimated the spin of the BH in LMC X-3.

In $\S 2$ we describe the continuum-fitting method and summarize our results. In $\S 3$ we discuss some of the remaining uncertainties in our disk model. We conclude with a brief discussion in $\S 4$. 
TABLE 1

Spin Estimates of Stellar-Mass Black Holes

\begin{tabular}{lccl}
\hline BH Binary System & $M / M_{\odot}$ & $a_{*}$ & Reference \\
\hline 4U 1543-47 & $8.4-10.4$ & $0.7-0.85$ & Shafee et al. (2006) \\
\hline GRO J1655-40 & $6.0-6.6$ & $0.65-0.8$ & Shafee et al. (2006) \\
\hline GRS 1915+105 & $10-18$ & $0.98-1$ & McClintock et al. (2006) \\
\hline LMC X-3 & $5.9-9.2$ & $<0.26$ & Davis et al. (2006) \\
\hline \hline
\end{tabular}

\section{THE METHOD}

A definite prediction of general relativity is the existence of an innermost stable circular orbit (ISCO) for a test particle orbiting a $\mathrm{BH}$. Once a particle is inside this radius, no stable orbits are available and the particle plunges into the hole. Gas in a geometrically thin accretion disk has negligible pressure support in the radial direction and behaves for many purposes like a test particle. Thus, the gas spirals in slowly (as a result of viscosity) through a series of nearly circular orbits until it reaches the ISCO, and it then plunges suddenly into the BH. In other words, the disk is effectively truncated at the ISCO. Therefore, if we can measure the radius of the disk inner edge, we will obtain the radius of the ISCO, $R_{\mathrm{ISCO}}$.

Since the dimensionless ratio, $\xi \equiv R_{\mathrm{ISCO}} /\left(G M / c^{2}\right)$, is a monotonic function of the $\mathrm{BH}$ spin parameter $a_{*}$ (Fig. 1), knowing its value allows us immediately to infer the $\mathrm{BH}$ spin parameter $a_{*}$. The variations in $R_{\text {ISCO }}$ are large - fully a factor of 6 as $a_{*}$ increases from 0 to unity - which implies that we should in principle be able to estimate $a_{*}$ with good precision.

In our work on BH X-ray binaries, we estimate the radius of the inner edge of the disk by fitting the X-ray continuum spectrum. For this purpose, we use the idealized thin disk model of Novikov \& Thorne (1973, hereafter NT model) which describes an axisymmetric radiatively-efficient accretion flow. For a given $\mathrm{BH}$ mass $M$, mass accretion rate $\dot{M}$ and $\mathrm{BH}$ spin parameter $a_{*}$, the NT model has a precise prediction for the profile of the radiative flux $F_{\text {disk }}(R)$ emitted by the disk as a function of radius $R$. Moreover, the accreting gas is optically thick, and the emission is thermal and blackbody-like, making it relatively straightforward to compute the spectrum of the emission (but see \$3.2). Therefore, by analyzing the spectrum of the disk radiation and combining it with knowledge of the distance $D$ to the source, the mass $M$ of the $\mathrm{BH}$, and the inclination $i$ of the disk, we can estimate $R_{\mathrm{ISCO}}$ and thereby obtain $a_{*}$.

For a full description of the mechanics of our current continuum-fitting methodology, we refer the reader to $\S 4$ in McClintock et al. (2006). In brief, we first select rigorouslydefined thermal-state X-ray data (McClintock \& Remillard 2006). This is because a BH accretion disk in the thermal state is likely to be very well described by the NT disk model. We fit the broadband X-ray continuum spectrum using the NT model, combined with an advanced treatment of spectral hardening (\$3.2). We also include (see Li et al. 2005) self-irradiation of the disk ("returning radiation"), limb darkening, gravitational 


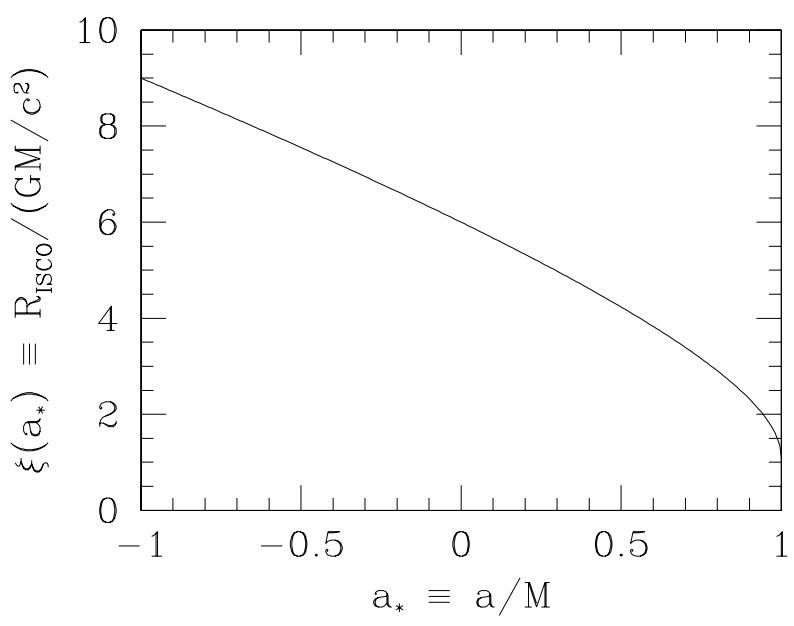

FIGURE 1. Shows the dependence of the quantity, $\xi=R_{\mathrm{ISCO}} /\left(G M / c^{2}\right)$, on the BH spin parameter, $a_{*} \equiv a / M=c J / G M^{2}$ (Shapiro \& Teukolsky 1983). The spin parameter is restricted to the range $-1 \leq$ $a_{*} \leq 1$; negative values correspond to the $\mathrm{BH}$ counter-rotating with respect to the particle orbit.

and Doppler redshifts, deflection of photon trajectories in the metric of the $\mathrm{BH}$, and the effect of a torque of any magnitude at the inner edge of the disk, although our published results are based on zero torque (see \$3.1).

Using the disk model, we fit directly for the two parameters of interest: the spin $a_{*}$ and the mass accretion rate $\dot{M}$. Using the known radiative efficiency of the NT disk model for a given $a_{*}$, and the fitted value of $\dot{M}$, we compute for each independent spectral observation the Eddington-scaled luminosity, $L / L_{\mathrm{Edd}}$, and consider only those observations for which $L / L_{\mathrm{Edd}} \leq 0.3$, which corresponds to disk thickness $H / R \leq 0.1$ (see McClintock et al. 2006). Finally, we present our results in the form of plots of $a_{*}$ versus $\log \left(L / L_{\text {Edd }}\right)$.

As an example, Figure 2 shows our results for GRS 1915+105 (McClintock et al. 2006). Over the luminosity range $L / L_{\mathrm{Edd}} \leq 0.3$, the data are consistent with a single value of $a_{*}$ close to unity. Allowing for statistical errors and uncertainties in the input values of $M, i$ and $D$, we estimate $a_{*}$ to lie in the range $0.98-1$ (Table 1). For luminosities closer to Eddington, the $a_{*}$ estimates obtained with our method are lower. We note that Middleton et al. (2006) obtained a very different spin estimate for GRS 1915+105 than we did because they relied exclusively on high luminosity data, which we argue is unreliable for the determination of spin (\$3.1).

In our method, we assume that the inner regions of the disk are aligned with the equatorial plane of the spinning BH. This is likely to be true because of the effects of frame-dragging and precession. In addition, we assume that the disk plane is aligned with the orbital plane of the binary system. This assumption allows us to estimate the inclination angle $i$ of the disk from the inclination of the binary orbit (which can be obtained from measurements of ellipsoidal modulation in the optical/infrared light curve). Thus, in effect, we assume that the $\mathrm{BH}$ spin axis is approximately aligned with 


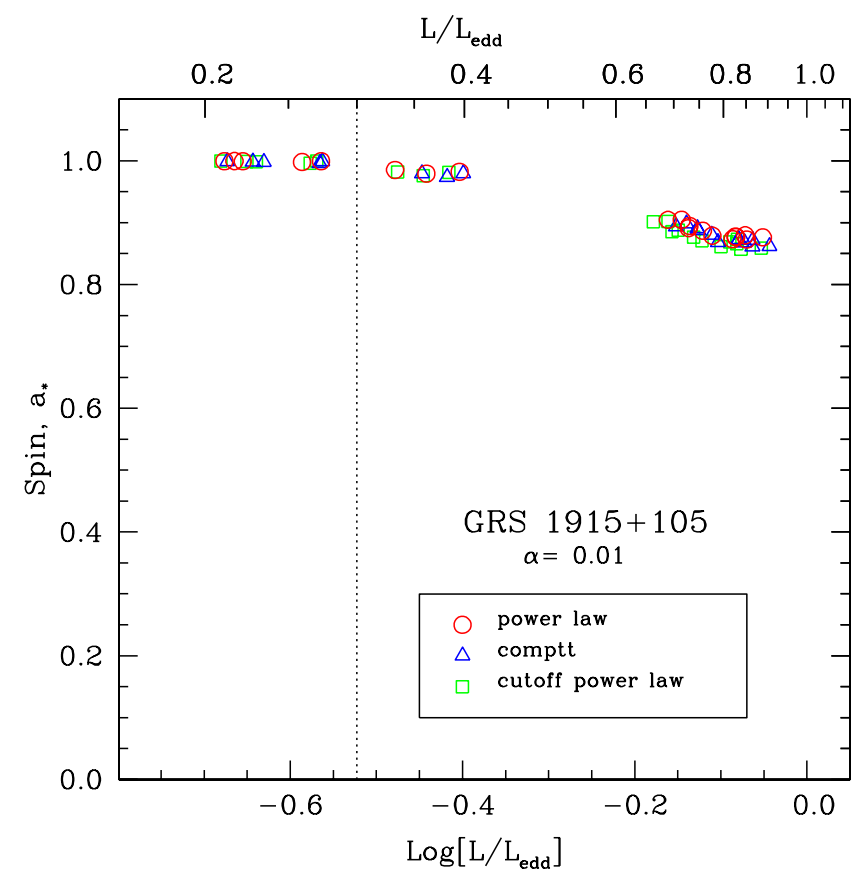

FIGURE 2. Shows the estimated spin parameter $a_{*}$ of the BH in GRS $1915+105$, as a function of the Eddington-scaled luminosity $L / L_{\text {Edd }}$. The spectral data were analyzed using the model KERRBB 2 (Li et al. 2005; McClintock et al. 2006) combined with three different models of the high energy Comptonized radiation (shown by different symbols). For $L / L_{\mathrm{Edd}}<0.3$ (to the left of the vertical dotted line), all the estimates of $a_{*}$ are consistent with a value nearly equal to unity. The result is insensitive to the precise Comptonization model used in the analysis.

the orbital angular momentum of the binary. There is no strong contrary evidence to this assumption, despite the often-cited examples of GRO J1655-40 and SAX J1819.32525 (see $\$ 2.2$ in Narayan \& McClintock 2005). GRS 1915+105 is a special case. For this source, we obtain the inclination angle of the disk from the known orientation of the radio jets, which are presumably perpendicular to the disk.

\section{THEORETICAL ISSUES}

Even with the best data, and assuming no errors in our estimates of disk inclination, the results we obtain are still only as good as the model we use to fit the observations. Here we discuss two crucial issues.

\subsection{Reliability of the Model of $F_{\text {disk }}(R)$}

The NT model on which we base our analysis assumes a thin accretion disk with a steady mass accretion rate. Because we limit ourselves to luminosities below $0.3 L_{\text {Edd }}$, the disks we study have $H / R \leq 0.1$ (McClintock et al. 2006) and are evidently thin. A 

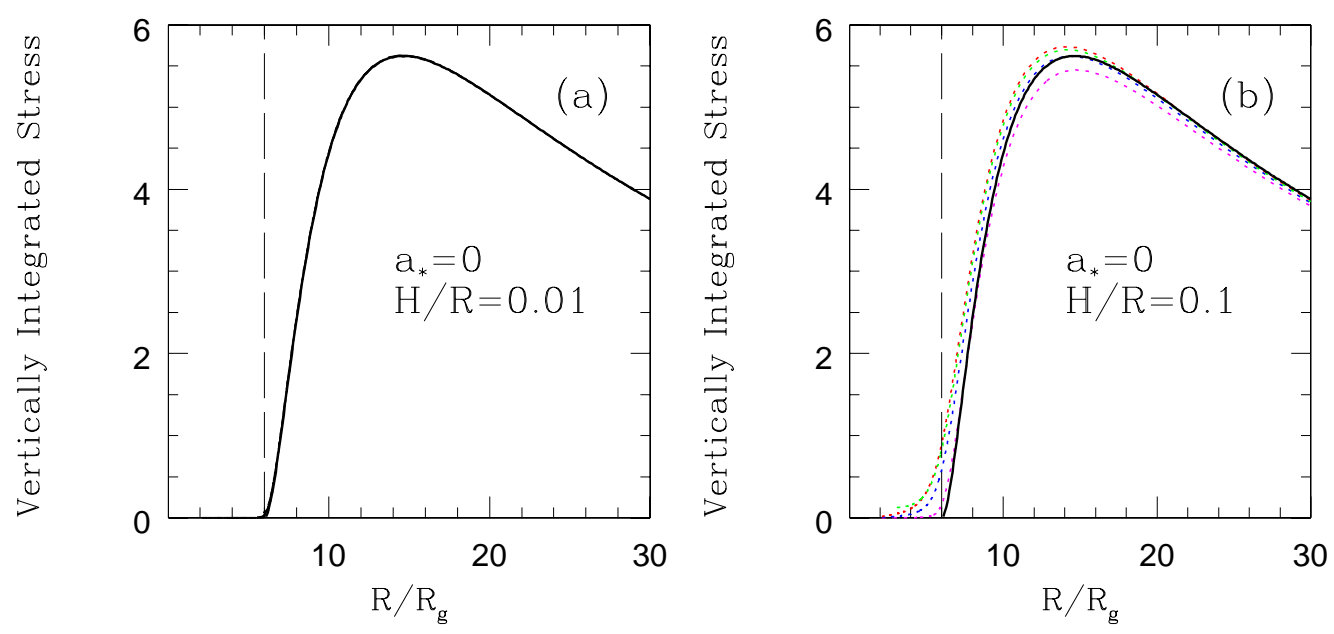

FIGURE 3. Shows the vertically integrated viscous stress profiles of a series of hydrodynamic models with $H / R=0.01$ (left) and $H / R=0.1$ (right). The $\mathrm{BH}$ is taken to be non-spinning, which corresponds to $R_{\mathrm{ISCO}} / R_{g}=6$, where $R_{g}=G M / c^{2}$. In each panel, the solid line corresponds to the NT model (more precisely, the equivalent of the NT model for the Paczyński-Wiita potential used in these Newtonian calculations) and the dotted lines correspond to hydrodynamic models for various choices of $\alpha$ (see Shafee et al. 2007 for details). It is seen that the hydrodynamic models agree very well with the NT model for these disk thicknesses.

thin accretion disk has the remarkable feature that the vertically integrated viscous shear stress at any radius $R$ is uniquely determined by $M, \dot{M}$ and $a_{*}$, and is independent of the details of the viscosity (Shakura \& Sunyaev 1973; NT). The energy dissipation rate per unit area is simply the product of the stress and the local gradient of the Keplerian velocity profile. Thus, the energy dissipation profile is very well determined. Further, the dissipated energy is immediately radiated (since a thin disk is radiatively efficient), so we can calculate $F_{\text {disk }}(R)$ precisely. All this means that the profile of $F_{\text {disk }}(R)$ that we use for spectral fitting is likely to be quite accurate.

There is, however, one important caveat, viz., the NT model assumes that the shear stress vanishes at the ISCO. To test the validity of this assumption, we have calculated numerical viscous-hydrodynamic thin disk models using height-integrated differential equations with $\alpha$-viscosity and the Paczyński-Wiita (1980) pseudo-Newtonian potential (Shafee, Narayan \& McClintock 2007). The key feature of our work is that we do not impose any boundary condition at the ISCO. Instead, we self-consistently solve for the position of the sonic radius, where the gas makes the transition from subsonic viscous inflow to supersonic free-fall into the $\mathrm{BH}$. The differential equations provide natural boundary conditions at this radius.

Figure 3 shows profiles of the height-integrated viscous stress for a number of hydrodynamic disk models with $H / R=0.01$ and 0.1 , and compares them to the profile predicted by the idealized NT model (the solid line). We see that the hydrodynamic models agree very well with the NT model. This means that our results on BH spin are likely to be reliable so long as $H / R \leq 0.1$, i.e., $L / L_{\text {Edd }} \leq 0.3$. Afshordi \& Paczyński 


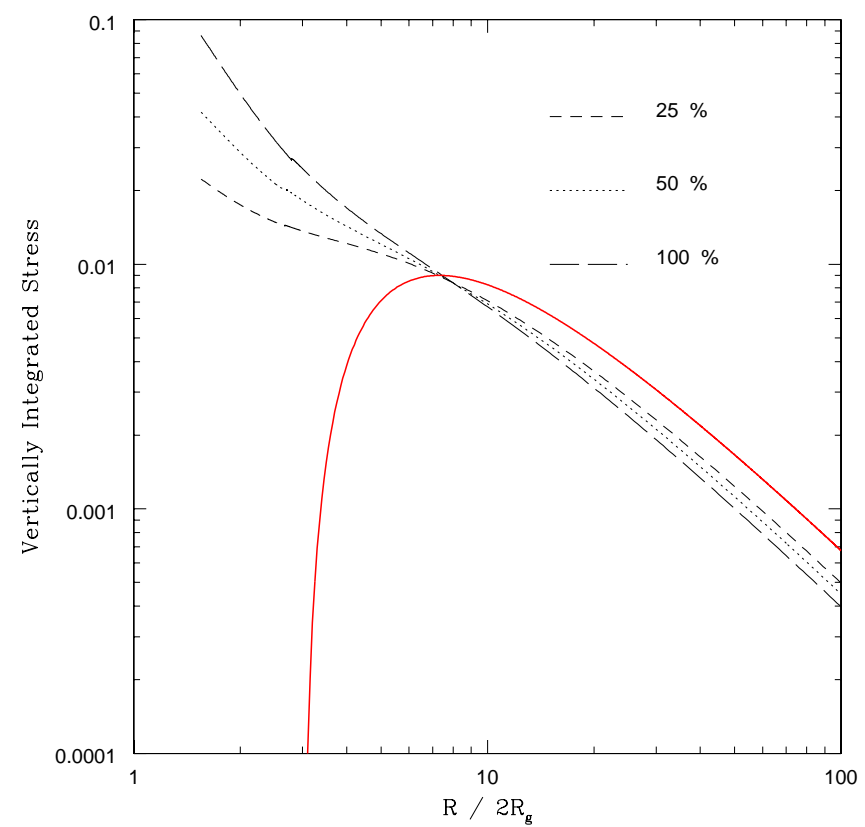

FIGURE 4. Similar to Fig. 3, but for thicker disks (from Shafee et al. 2007). The disk thickness is adjusted by varying the fraction of the dissipated energy that is retained in the gas $(25 \%, 50 \%$ and $100 \%$ for the three models shown by dashed and dotted lines). Notice the large deviations between these thick disk models and the NT model (solid line).

(2003) had previously reached a similar conclusion.

Figure 4 shows in contrast what happens for thicker, more luminous disks. The shear stress profiles in these models deviate enormously from the NT model. Clearly, we cannot hope to obtain reliable estimates of $\mathrm{BH}$ spin under these conditions. This is precisely what we find for GRS 1915+105. In Fig. 2, the points at higher luminosities are increasingly discrepant from the results we trust, viz., those that correspond to luminosities below $0.3 L_{\text {Edd. }}$. In our view, the results of Middleton et al. (2006) on GRS $1915+105$ are not reliable because they focused exclusively on high luminosity data.

While the results shown in Figs. 3 and 4 are very encouraging, we note that our calculations correspond to a hydrodynamic model, whereas real disks doubtless have strong magnetic fields. How well do magnetized thin disks respect the zero-stress condition at the ISCO? This is presently an open question, since most of the MHD work to date (Krolik 1999; Gammie 1999; Hawley \& Krolik 2002) has focused on thick disks. Interestingly, the stress profiles we find for thick (radiatively inefficient) hydrodynamic disks (Fig. 4) are quite similar to the profile obtained by Krolik \& Hawley (2002) from their 3D MHD simulation of a radiatively inefficient disk (see their Fig. 10). MHD simulations of thin $(H / R \leq 0.1)$ disks are highly desirable to confirm the hydrodynamic results shown in Fig. 3. 


\subsection{Spectral Hardening}

Even when an accretion disk is in the thermal state, it does not radiate as a perfect blackbody. Electron scattering and Comptonization modify the emerging spectrum. This effect was first considered by Shimura \& Takahara (1995), who showed that to a good approximation the emerging spectrum can be desribed by means of a spectral hardening factor $f$. That is, the spectrum retains the shape of a blackbody, but the temperature $T$ of the radiation is related to the radiative flux by $F_{\text {disk }}=\sigma(T / f)^{4}$, where $\sigma$ is the Stefan-Boltzmann constant.

In our work, we use the state-of-the-art spectral models of Davis et al. (2005) to compute tables of $f$ versus $L / L_{\text {Edd }}$ for each BH mass $M$ and disk inclination $i$ of interest. A feature of Davis's work is that he includes metal opacities, whereas Shimura $\&$ Takahara considered only a light element atmosphere. The metals generally reduce the amount of spectral hardening.

Because of the inclusion of the detailed disk atmosphere computations of Davis, we believe the spectral model we use for our BH spin work is reliable. The thermal state has negligible energy in an external corona, so there are unlikely to be any significant surface effects that might modify the spectrum. The one remaining issue has to do with the vertical density structure in the disk. The Davis model assumes vertical hydrostatic equilibrium and uses a general equation of state that includes both gas and radiation pressure. For this case, the model is likely to be very accurate. Astrophysicists have considerable experience in carrying out such calculations through decades of work on stellar atmospheres. However, recent radiation MHD simulations (Blaes et al. 2006) suggest that magnetic support might be significant near the surface of an accretion disk. This would reduce the gas density in the atmosphere and cause additional hardening of the spectrum. Ongoing numerical simulations should clarify the situation.

\section{DISCUSSION}

In order to model the ways that an accreting BH can interact with its environment, one must know its spin. Many studies have suggested a link between relativistic jets and BH spin (e.g., Meier 2003; McKinney \& Gammie 2004; Hawley \& Krolik 2006), but observational confirmation will be possible only when we have measured the spins of a reasonable sample of BHs. Likewise, measurements of spin are important for testing collapsar models of Gamma-Ray Burst sources (Woosley 1993; MacFadyen \& Woosley 1999) and for understanding binary evolution and BH formation in general (e.g., Brown et al. 2007). In this context we note that the high spins we have measured for three of the four BHs listed in Table 1 were very likely imparted to these BHs during the process of their formation (see $\$ 6.2$ in McClintock et al. 2006). Knowledge of BH spin is also crucial for the development of gravitational-wave astronomy and for models of quasiperiodic oscillations in BH X-ray binaries (Török et al. 2005).

We are presently working on two other systems, M33 X-7 and XTE J1550-564, and we expect to report the spins of these BHs within the next few months. Over the next 3-4 years, we anticipate epanding the sample of spin estimates to about a dozen. 


\section{REFERENCES}

Afshordi, N., and Paczyński, B., ApJ, 592 354-367 (2003).

Blaes, O. M., Davis, S. W., Hirose, S., Krolik, J. H., and Stone, J. M., ApJ 645, 1402-1407 (2006).

Brown, G. E., Lee, C.-H., Moreno-Mendez, E., and Walter, F. M., astro-ph/0612461 (2007).

Davis, S. W., Blaes, O. M., Hubeny, I., and Turner, N. J., ApJ 621, 372-387 (2005).

Davis, S. W., Done, C., and Blaes, O. M., ApJ 647, 525-538 (2006).

Gammie, C. F., ApJ 522, L57-L60 (1999).

Hawley, J. F., and Krolik, J. H., ApJ 566, 164-180 (2002).

Hawley, J. F., and Krolik, J. H., ApJ 641, 103-116 (2006).

Krolik, J. H., ApJ 515, L73-L76 (1999).

Krolik, J. H., and Hawley, J. F., ApJ 573, 754-763 (2002).

Li, L.-X., Zimmerman, E. R., Narayan, R., and McClintock, J. E., ApJS 157, 335-370 (2005).

MacFadyen, A. I., and Woosley, S. E., ApJ 524, 262-289 (1999).

McClintock, J. E., and Remillard, R. A., "Black Hole Binaries," in Compact Stellar X-ray Sources, edited by W. Lewin and M. van der Klis, Cambridge Univ. Press, 2006, pp. 157-213.

. McClintock, J., E., Shafee, R., Narayan, R., Remillard, R. A., Davis, S. W., and Li, L.-X., ApJ 652, 518-539 (2006).

. McKinney, J. C., and Gammie, C. F., ApJ 611, 977-995 (2004).

. Meier, D. L., New Astron. Rev. 47, 667-672 (2003).

- Middleton, M., Done, C., Gierliński, M., and Davis, S. W., ApJ 373, 1004-1012 (2006).

Narayan, R., and McClintock, J. E., ApJ 623, 1017-1025 (2005).

. Novikov, I. D., and Thorne, K. S., "Black Hole Astrophysics," in Blackholes, edited by C. DeWitt and B. DeWitt, Gordon \& Breach, 1973, pp. 343-450.

Orosz, J. A., McClintock, J. E., Narayan, R., Bailyn, C. D., Hartman, J. D., Macri, L., Liu, J., Pietsch, W., Remillard, R. A., Shporer, A., and Mazeh, T., Nature in press (2007).

Paczyński, B., \& Wiita, P. J., A\&A 88, 23-31 (1980).

- Shafee, R., McClintock, J. E., Narayan, R., Davis, S. W., Li, L.-X., and Remillard, R. A., ApJ 636, L113-L116 (2006).

Shafee, R., Narayan, R., and McClintock, J. E., ApJ submitted (2007) arXiv:0705.2241.

Shakura, N. I., and Sunyaev, R. A., A\&A 24, 337-355 (1973).

Shapiro, S. L., and Teukolsky, S. A., Black Holes, White Dwarfs, and Neutron Stars, Wiley (1983).

Shimura, T., and Takahara, F., ApJ 445, 780-788 (1995).

Török, G., Abramowicz, M. A., Kluz'niak, W., and Stuchlik, Z., A\&A 436, 1-8 (2005).

Woosley, S. E., ApJ 405, 273-277 (1993).

Zhang, S. N., Cui, W., and Chen, W., ApJ 482, L155-L158 (1997). 